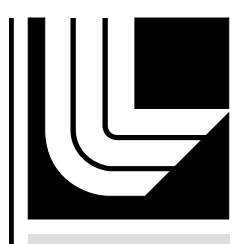

LA W REN CE LIVERM ORE N A TIO NAL LABORATORY

FY13 TPC Initial Data Run

M. Heffner

October 7, 2013 
This document was prepared as an account of work sponsored by an agency of the United States government. Neither the United States government nor Lawrence Livermore National Security, LLC, nor any of their employees makes any warranty, expressed or implied, or assumes any legal liability or responsibility for the accuracy, completeness, or usefulness of any information, apparatus, product, or process disclosed, or represents that its use would not infringe privately owned rights. Reference herein to any specific commercial product, process, or service by trade name, trademark, manufacturer, or otherwise does not necessarily constitute or imply its endorsement, recommendation, or favoring by the United States government or Lawrence Livermore National Security, LLC. The views and opinions of authors expressed herein do not necessarily state or reflect those of the United States government or Lawrence Livermore National Security, LLC, and shall not be used for advertising or product endorsement purposes.

This work performed under the auspices of the U.S. Department of Energy by Lawrence Livermore National Laboratory under Contract DE-AC52-07NA27344. 


\title{
FY13 TPC Initial Data Run
}

\author{
Mike Heffner, LLNL
}

27Sept2013 


\begin{abstract}
This is a report of the fission TPC project for the fiscal year 2013. Included is a description of the experimental work and analysis of the data from the experiment. This report is closeout criterion for the TPC L2: "Report describing results of initial data run, not including complete analysis."
\end{abstract}




\section{Introduction}

The fission Time Projection Chamber was designed to measure fission cross sections with unprecedented accuracy. While sensitivity studies have shown that the $\mathrm{Pu}-239$ fission cross section needs to be known to about $1 \%$ for defense applications, existing techniques are limited to 3-5\% uncertainties. The TPC takes advantage of particle tracking capabilities to reduce systematic uncertainties to reach that $1 \%$ requirement.

Some key features of the TPC needed to optimize accuracy were demonstrated in the 2013 LANSCE run cycle. By having half of the TPC instrumented we were able to measure full fission fragment tracks in a neutron beam experiment, demonstrating the tracking capability needed to reduce systematic uncertainties associated with the fission fragment tracks. A second important demonstration was the ability to accurately measure neutron time-offlight. This is another key element in achieving high precision in neutron-induced fission cross sections, and was for the first time successfully implemented with the TPC.

\section{Scope}

The scope of this report is the fission TPC data run at LANSCE starting 23rd August 2012 and ending 2nd Feb 2013 (the FY13 data run) and the initial analysis of the data collected in this period. The TPC has just recently reached another milestone of double sided operation, a fully instrumented TPC. This is a very recent development and is not included in the scope of this L2 milestone document.

\section{Hardware Setup}

The TPC with one side fully instrumented was installed at LANSCE prior to the FY13 run period and was run throughout. The run period consisted of 5 runs each varying in length from 12 to 29 days, see figure 1 . The TPC was operated during all of these runs and the data collected has been stored in a large capacity tape storage located at LLNL. The down period between runs was used to do maintenance on the TPC and perform task such as switching targets.

The TPC was loaded with $1 / 2$ of the electronics providing $2 \pi$ coverage of the fission events for the first time. This involved the construction and installation of 2976 channels of electronics, each channel a charge preamp and $62.5 \mathrm{MHz}$ waveform digitizer. Figure 2 shows the fully instrumented TPC and figure 3 shows the TPC located in the LANL beam line. This configuration was run for the entire LANSCE run period. The $2 \pi$ coverage provided the first dataset that we could start looking at cross sections and the details of the TPC performance in detail because we no longer have edge effects from the missing sectors.

Also the time of flight (TOF) was first made functional this run cycle. This system provides a high speed timing mark of when the fission occurs and an overall energy deposited in the TPC gas volume. This mark is recored in the TPC data stream along with matching accelerator timing marks. This TOF serves two important functions. The first is that it 


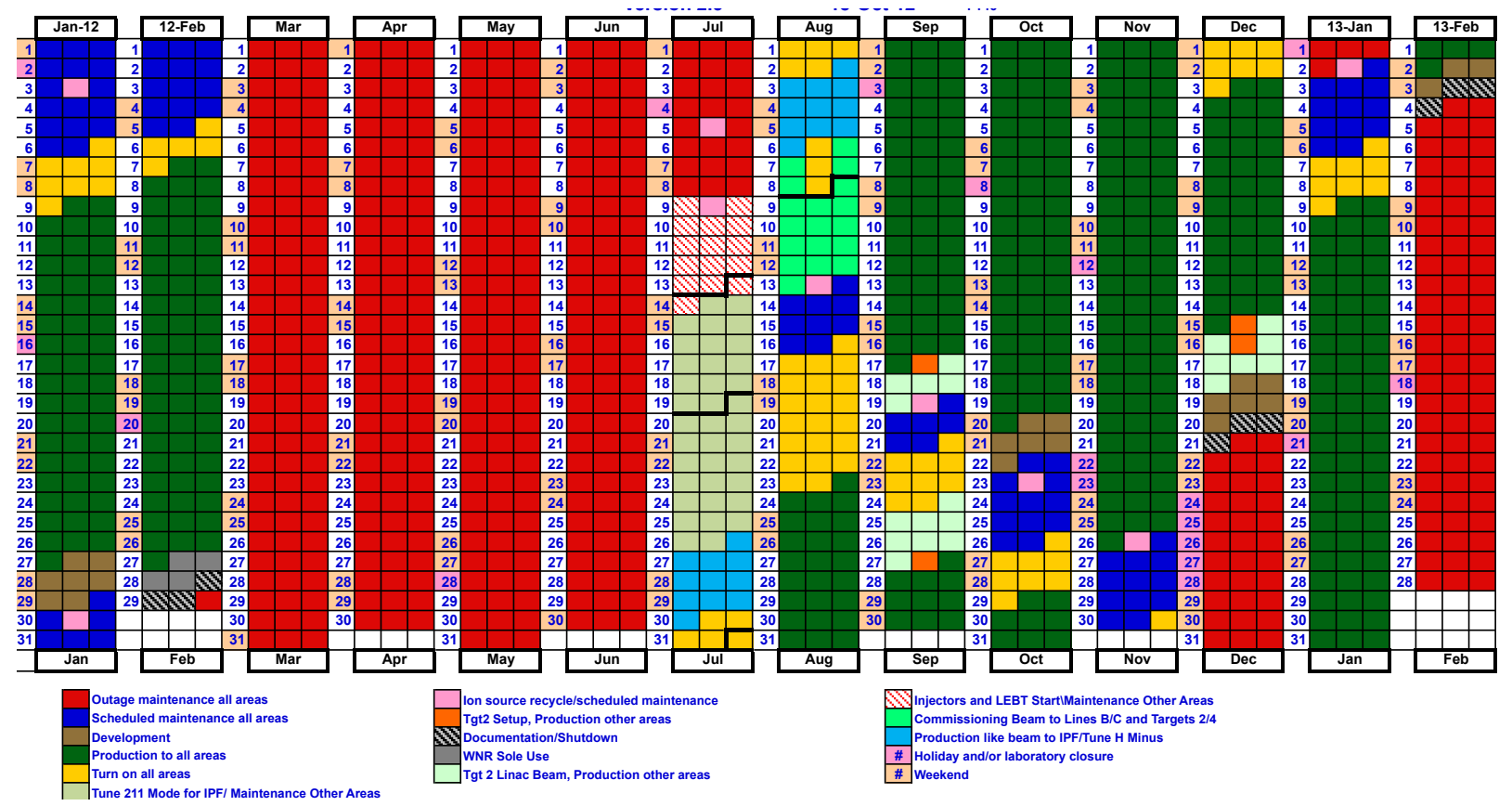

Figure 1: The FY13 LANSCE run schedule. The areas in green depict the planned beam time for this run period.

is compared with a signal from the accelerator to determine the time of flight of a neutron produced at the primary target that causes a fission in the TPC. This timing is then converted into an energy which is used to determine the energy of the neutron that produced a particular fission. From this an energy dependent cross section is developed. The second function is a start signal for the TPC. Since the TPC derives the z-direction from the drift time of electrons in the gas, a start time is needed to correctly place the track in $\mathrm{z}$.

The hardware that provides the TOF consists of a few parts. The first is a low-noise high-speed transimpedance current amplifier placed as close a possible to the cathode. In these runs we used a modified Cermat amplifier on the unused side of the TPC in the gas volume. (With one sided operation the second side was not used and a easy place for the test amplifier.) This amplifier produced good results and the next task is to optimize this amplifier by lowering the noise and pickup and to design an amplifier that does not interfere with the second side once is it in operation in the next run period.

Deploying a detector into a new environment usually comes with some difficulties and the TPC had to solve a significant one in this run period. It was discovered that the TPC would frequently spark and trip HV when the beam was on. We could still take data, but it was a significant burden to the project as it took a lot of time to reset and each spark typically killed a preamp channel. Spark protection was added to the preamps to prevent damage to them as one mitigation of the problem. We also looked to understand the source and it seems that a likely candidate is the (n,n') interaction on the argon and carbon in the 


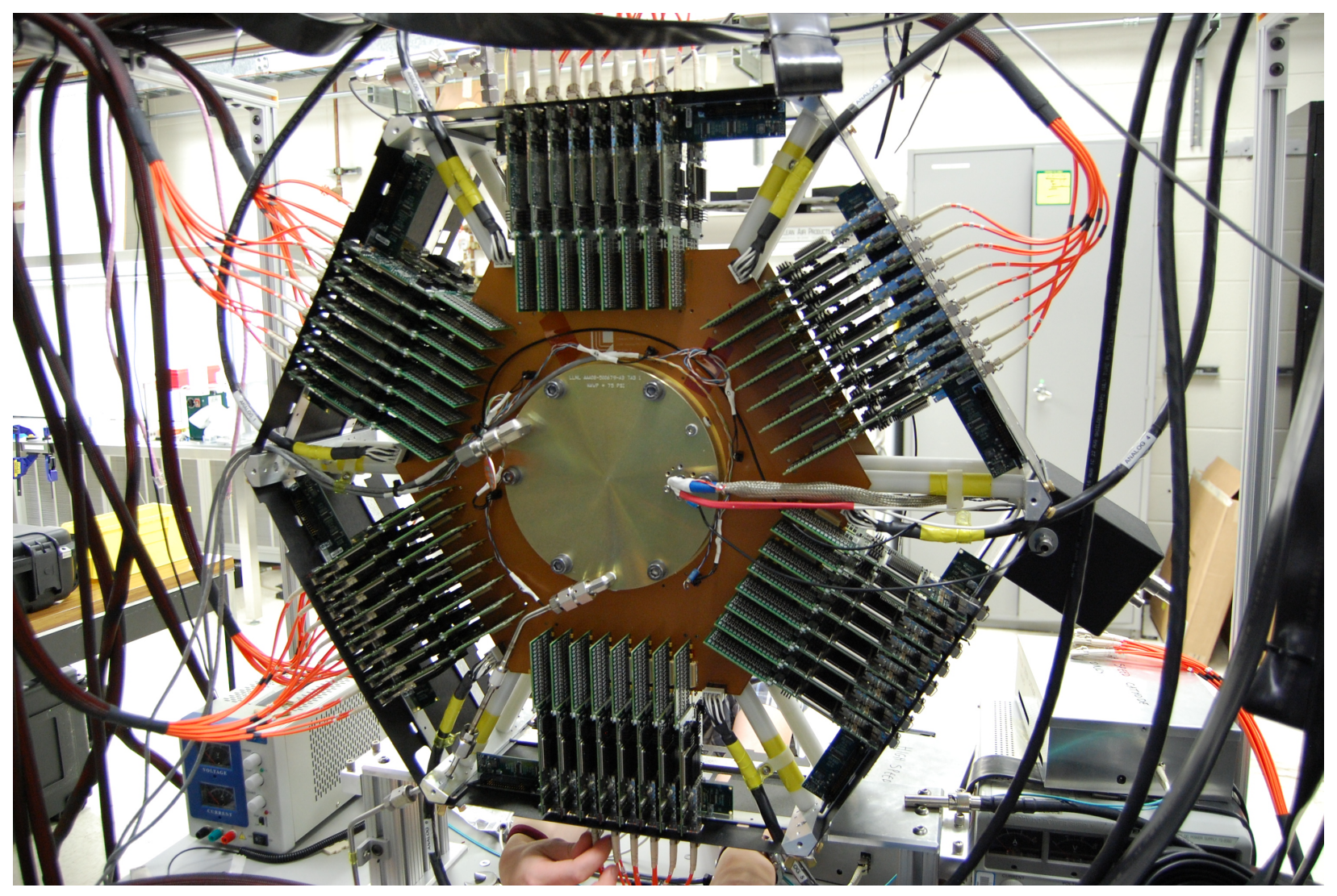

Figure 2: The TPC loaded with half of the electronics, 2976 channels.

gas. These highly ionizing nuclear recoils move only a short distance and produce a high charge density. This ionization density is similar to fission fragments, but there is one key difference in that they can be produced anywhere in the gas whereas the fission fragments are produced nearer to the cathode and diffuse on the way to the micromegas. The (n,n') recoils that are produced near or in the micromegas have a very high charge denisty and there for could exceed the limits of the micromegas causing a trip.

With this theory we simulated a number of gasses looking for a replacement that maintaned the good properties of P10 but had better quenching. We arrived at a mixture of $95 \%$ argon $5 \%$ isobutane. This is a very stable gas and we no longer have any HV trips and we can even achieve a little higher gain than with P10. There is still a little more work to make sure this mixture does not have gain loss at high rates, but at least the sparking has been solved. 


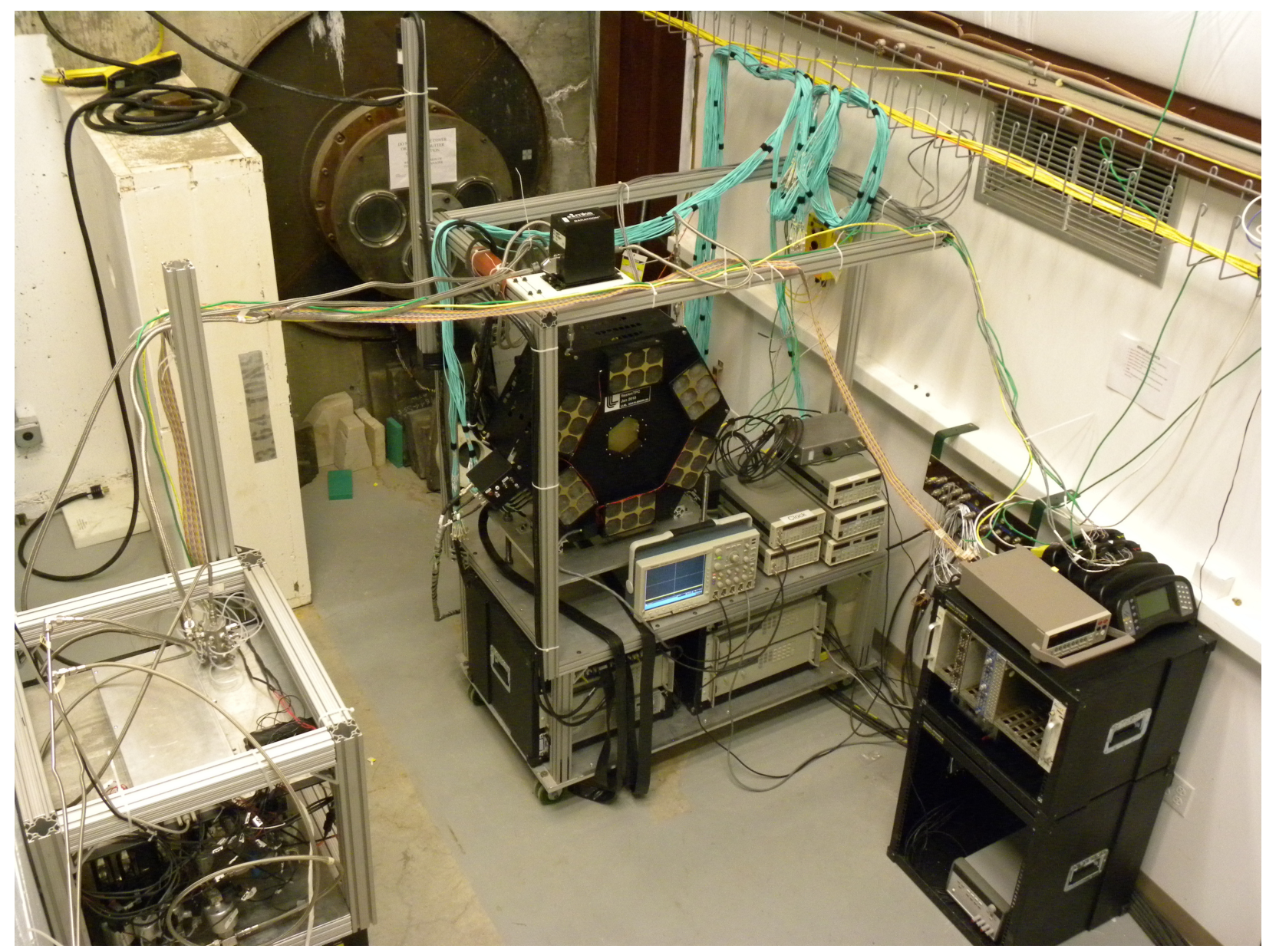

Figure 3: A picture of the TPC located in the beam line at LANL. The electronics have not been installed this picture, but the gas system, power supplies and other auxiliary equipment is visible.

\section{Targets}

The targets are the actinide materials that are irradiated with the neutron beam and the resulting interaction are studied with the TPC. These are obviously critical to the experiment and are exclusively constructed at Oregon State. The target holder is a $4 \mathrm{~cm}$ diameter aluminum disk and the material under study is located at the inner $2 \mathrm{~cm}$. Some targets have a deposit at the center of a solid target holder disk and in some cases we have cut away the aluminum and placed a thin backing material for the inner $2 \mathrm{~cm}$ on which the target materiel is placed. The thin backing materials are fragile and therefore require extra preparation and care, but also provide the ability to track both fragments from a fission event. The TPC has operated thin targets at LLNL with ${ }^{252} \mathrm{Cf}$ and is scheduled to operated with thin backing ${ }^{239} \mathrm{Pu}$ target in beam for the next run period. 


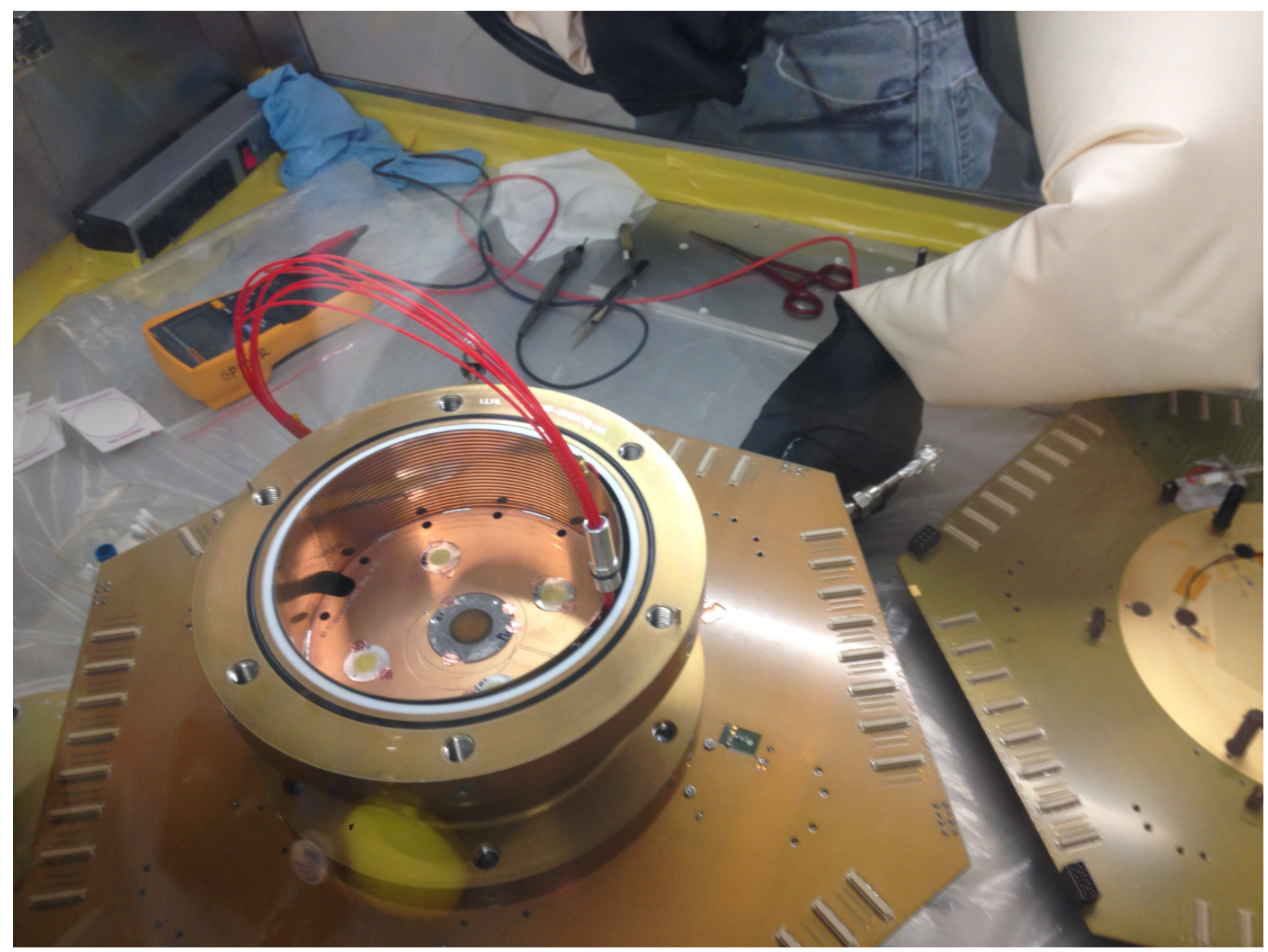

Figure 4: A ${ }^{239} \mathrm{Pu}$ target loaded into the TPC. The target is the center most black circle. The top side of the TPC is removed in order to see the target.

The targets are loaded at the very center of the TPC (see figure 4) with an active gas volume on either side providing nearly $4 \pi$ detection of the charged particles. The only particles not detected are lost because of interactions with the target, target holder or cathode which is only $1-2 \%$ of the total solid angle. Interactions with the cathode and target holder have been observed so the $1-2 \%$ loss is an overestimate since one can still measure them in some cases.

The ${ }^{239} \mathrm{Pu}$ target, because of the high activity $(\approx 1 \mathrm{MBq})$, is a minor health hazard and this combined with the fragile nature of the thin backings and the recent incident at Lujan an abundance of caution was exercised in the preparations for loading the TPC with the ${ }^{239} \mathrm{Pu}$ targets. Even though safe to handle on an open bench, it was decided to load the TPC targets in a glovebox, see figure 5. This required a number of preparation steps and bureaucratic hurdles which have been completed. The TPC is now routinely loaded in the 
glovebox and ${ }^{239} \mathrm{Pu}$ has been loaded a number of times.

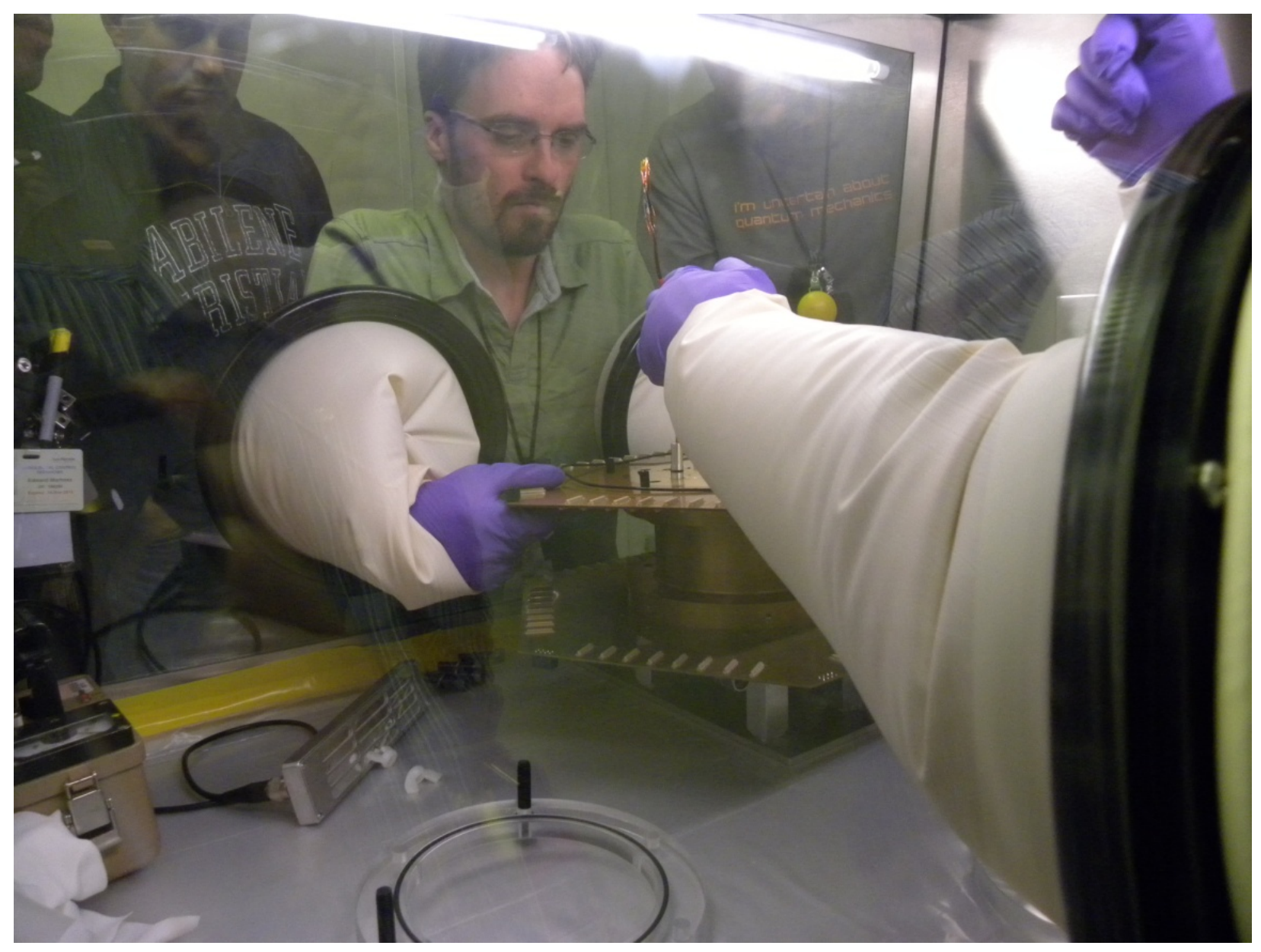

Figure 5: Example of glovebox work to load the targets into the TPC.

\section{Three targets were run in this run period}

- Two half circles, one of $238 \mathrm{U}$ and the other of $235 \mathrm{U}$ on thick $\mathrm{Al}$ backing This target was designed to study the tracking of the TPC, particle identification, target autoradiographs in situ, beam profiles and preliminary cross sections of 238U/235U.

- Two half circles, one of $239 \mathrm{Pu}$ and the other $235 \mathrm{U}$ on thick $\mathrm{Al}$ backing This target is very similar to the previous and extends the study to a high activity target and also a preliminary look at $239 \mathrm{Pu} / 235 \mathrm{U}$.

- A full circle of $239 \mathrm{Pu}$ on $\mathrm{Al}$ backing with plastic on the other side This target provided a greater level of alpha load on the system and also had a provision to look at the response of plasic on the other side of the target for the ratio of $239 \mathrm{Pu} / \mathrm{H}$. 


\section{Data Analysis}

The data analysis is taking a number of paths simultaneously to tackle all of the issues required to return a cross section study to meet the mid FY15 milestone. The descoping of parts of the FY15 milestone because of the NE funding loss have left the emphasis on particle identification and target/beam non-uniformities. These results will be the focus of the remainder of this report but it is important to reconize a number of analysis tasks have to be completed in order to meet this milestone.

- Channel to channel calibration

- Channel to channels threhsold setting

- TOF hardware calibration for each run

- TOF analysis including carbon filter calibration and the rise time parameteriztion of the cathode signal

- Time zero (t0) determination

- Drift speed calibration for each run

- Tracking: debug, work out diffusion effects and understand edge cases

- Speed analysis to process $100 \mathrm{~TB}$ of data in reasonable time

- Investigate and quantify the events unrelated to the signal of interest, such as $(\mathrm{n}, \mathrm{X}) \alpha$, spallation, (n,n') and others

- etc...

\section{Target uniformity}

The target uniformity is assessed with an autoradiograph and a strength of the TPC is the ability to do this in situ with the same coordinates and registration as the beam measurement. The autograiograph is performed by looking at the starting point of a spontaneous alpha from the target. A plot of track starting points for a $238 \mathrm{U} / 235 \mathrm{U}$ target without beam is shown in figure 6 . The two half circles are clearly visible. The the two actinides are distinguished because of the difference in the activity of the two deposits. This analysis still needs refinement in the tracking algorithm which provides the track start point and the time offset (t0) has not been properly corrected yet. Even with these items still in progress, the resolution of the TPC is rather good. 


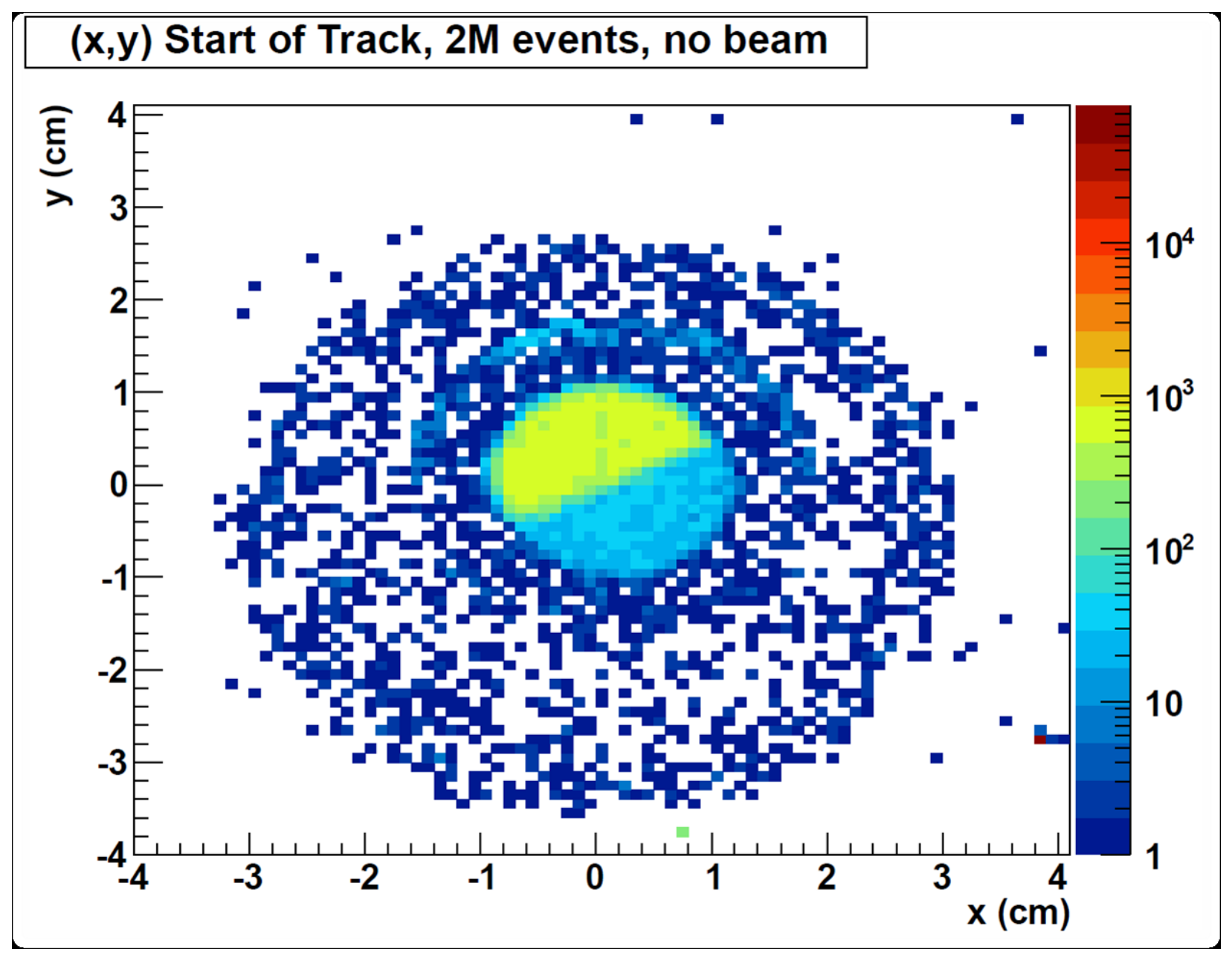

Figure 6: An autoradiograph of a $235 \mathrm{U} / 238 \mathrm{U}$ target showing the ability of the TPC to distinguish the location of target meaterial on the TPC cathode. The color indicates the density of tracks at any point.

\section{Beam uniformity}

The beam uniformity is the complement to the target uniformity and both need to be studied to return a high precision cross section. The beam uniformity is measured using interactions of the beam with the gas in the TPC. The relative beam intensity (uniformity) is best measured in the gas because the gas itself provides a very uniform target mass. The neutron beam interacts with the gas though a number of reactions such as (n,p) on the hydrogen in the isobutane in the gas. There are also (n,n') interactions with the argon and carbon also in the gas. Other reactions from materials containing the gas are naturally suppressed providing a nice method to measure the beam. A plot of these interactions in the TPC with the beam on is figure 7 .

Right away one can see a few interesting features. The first is that it is not a circular 


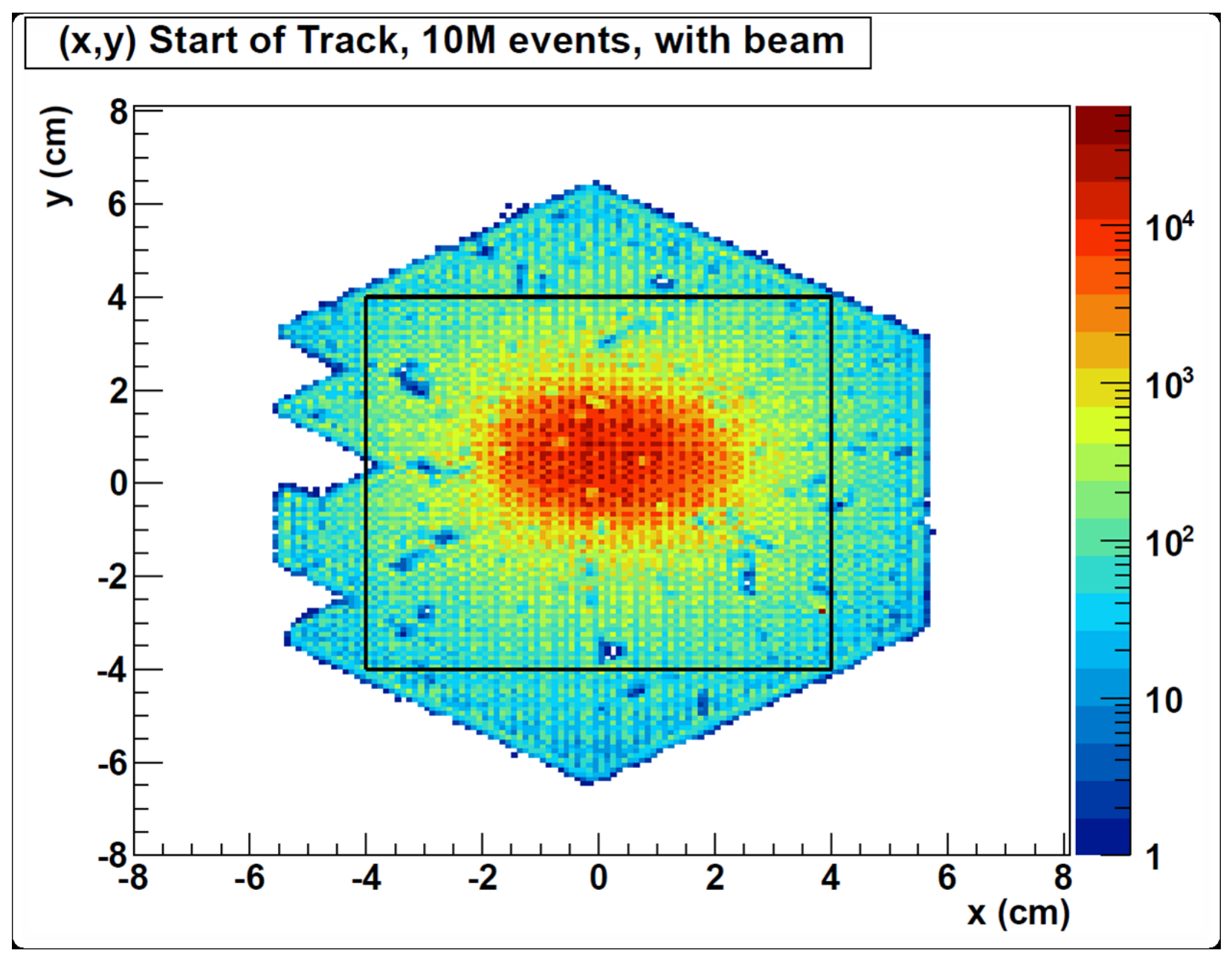

Figure 7: An image of track start locations in the TPC with beam. The track starts indicate the location of beam passing thought the TPC and thereby give the beam profile.

beam. This is because the beam collimation acts as a pin hole camera the the shape of the spallation target that generates the neutrons is visible. This was confirmed by measuring the slight angle of the image and comparing to the tilt in the spallation target. Another feature is that the intensity smoothly varies which makes it easier to work with and does not require as fine grained resolution. The TPC is also capable of looking at some beam interactions as a function of energy and with this we could understand how the beam spot changes with energy which has not been done before.

\section{Particle identification (PID)}

The final analysis highlight is the particle identification which is extremely important for the separation of alphas from the fission fragments. Figure 8 shows the length of a track 
on the $\mathrm{y}$-axis and the energy of the track on the $\mathrm{x}$-axis for a run using a ${ }^{252} \mathrm{Cf}$ target. This analysis has also been made for targets in beam, but it is easier to see features in this simpler plot. Each feature in this plot represents a different particle or distortion of a particle and we now have a full understanding of each feature in the plot. We believe that particle identification may be one of the largest systematic errors in the current data and this plot and the understanding of it provide a path to fully understand the issues in this and similar measurements.

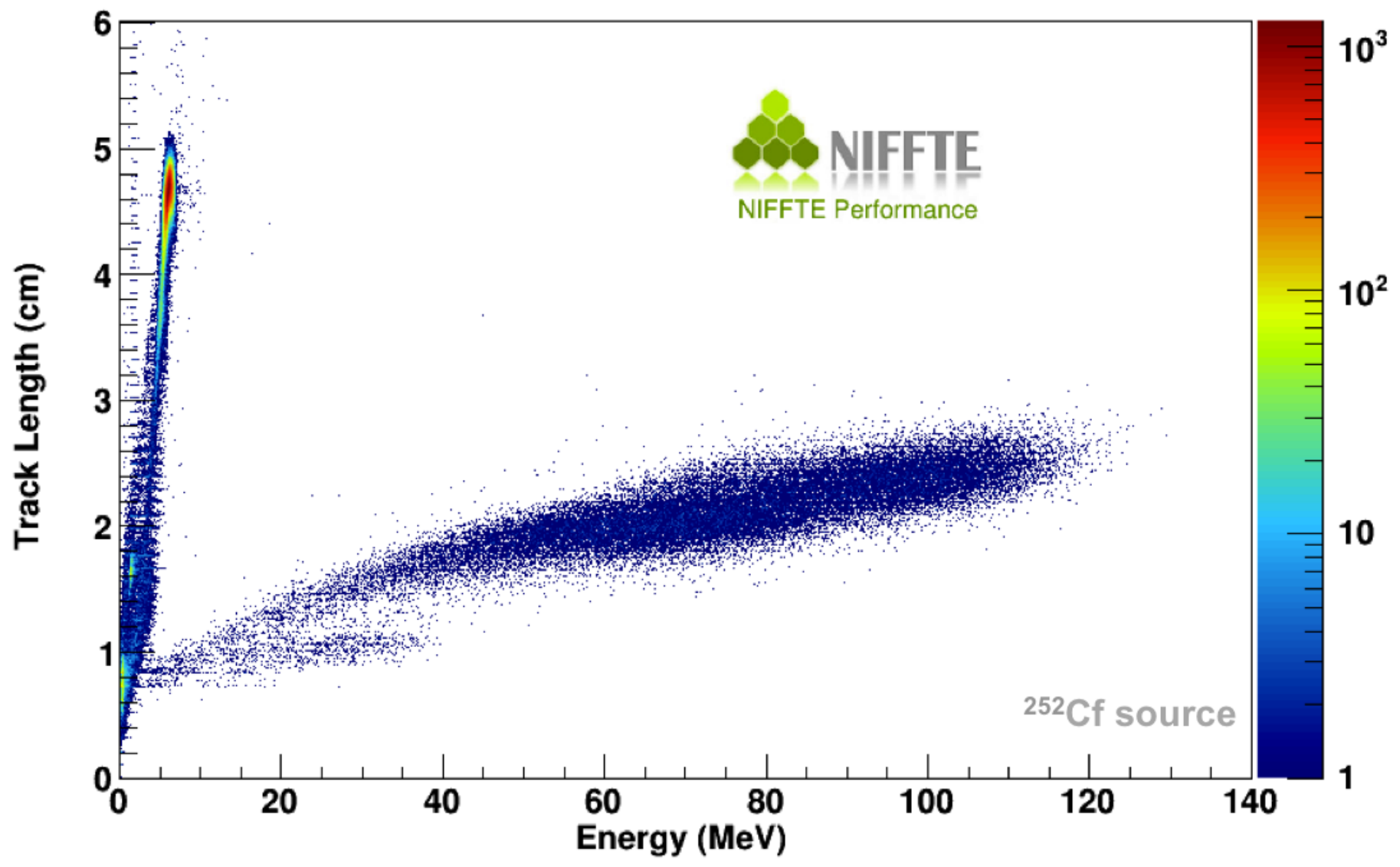

Figure 8: The resolving power of the TPC to distinguish different particles is evident in this plot of track length vs energy of the track. The vertical band is from alpha particles and the diagonal is from fission fragments.

It is useful to explain the PID in some detail as it illustrates the power of the TPC for these measurements. The vertical feature is the alpha particles from spontaneous decay the bulk of them located at about $5 \mathrm{~cm}$. If one studies this plot as a function of the angle of the track it is clear that the alphas leaving perpendicular to the target form the spot near $5 \mathrm{~cm}$ and the tail at lower track length is from alphas leaving the target and a shallow angle and 
thereby loosing energy in the target. Further down in track length there are two spots of higher intensity (light blue/green). These are tracks that hit the target holder and cathode and this is confirmed by looking at the location the track starts which is right at these diameters.

The band that is more centered is the fission fragments. There are two broad peaks in the center that are the light and heavy fragments. The tail dropping to the left is again framents that straggle in the target which is confirmed by looking at the angle of these tracks. The odd feature starting at about $20 \mathrm{MeV}$ up to $40 \mathrm{MeV}$ at a length of $1 \mathrm{~cm}$ is fission fragments hitting the cathode which was confirmed by looking at the starting location.

\section{Conclusion}

The TPC project has made great strides in completing the construction of the TPC, deploying it to a the LANSCE beam, taking data with the instrument and getting a good start on the analysis. All of the hard work in getting to this stage is now paying off and we are now starting to look at the details of fission in a TPC. 